\title{
Nano-Contact Problem with Surface Effects on Triangle Distribution Loading
}

\author{
Liyuan Wang*, Wei Han, Shanlin Wang, Lihong Wang, Yinping Xin
}

Regional Circular Economy key Laboratory of Gansu Higher Institutions; Department of Mathematics, Longqiao College of Lanzhou

University of Finance and Economics, Lanzhou, China

Email: ‘wly811220@163.com, talitha@sohu.com,wangshan1554517@163.com,wlh232x@sina.com,x.yp1987@163.com

How to cite this paper: Wang, L.Y., Han, W., Wang, S.L., Wang, L.H. and Xin, Y.P. (2016) Nano-Contact Problem with Surface Effects on Triangle Distribution Loading. Journal of Applied Mathematics and Physics, 4, 2047-2060.

http://dx.doi.org/10.4236/jamp.2016.411204

Received: August 29, 2016

Accepted: November 15, 2016

Published: November 18, 2016

Copyright $\odot 2016$ by authors and Scientific Research Publishing Inc. This work is licensed under the Creative Commons Attribution International License (CC BY 4.0).

http://creativecommons.org/licenses/by/4.0/

\begin{abstract}
This work presents a theoretical study of contact problem. The Fourier integral transform method based on the surface elasticity theory is adopted to derive the fundamental solution for the contact problem with surface effects, in which both the surface tension and the surface elasticity are considered. As a special case, the deformation induced by a triangle distribution force is discussed in detail. The results are compared with those of the classical contact problem. At nano-scale, the contributions of the surface tension and the surface elasticity to the stress and displacement are not equal at the contact surface. The surface tension plays a major role to the normal stress, whereas the shear stress is mainly affected by the surface elasticity. In addition, the hardness of material depends strongly on the surface effects. This study is helpful to characterize and measure the mechanical properties of soft materials through nanoindentation.
\end{abstract}

\section{Keywords}

Surface Tension, Surface Elasticity, Nano-Contact Problem, Fourier Integral Transforms Method, Triangle Distribution Force

\section{Introduction}

Nowadays, nanometer material and technology have been widely used in industrial and engineering fields. Many new nano-materials have been developed by utilizing the fact that materials begin to exhibit unique mechanical properties at nano-scale, which significantly differ from those at larger scale.

Nano-indention tests have been widely used to measure such mechanical properties of materials. For micro-nano solids with large surface-to-bulk ratio the significance of surfaces is likely to be important. Form the viewpoint of continuum mechanics, this 
difference can be described by such concepts as surface effects [1]. This is especially true for nano-scale materials or structures. In such cases, the surface tension and the surface elasticity play a critical role and thus have been adding its appeal to many researchers. For example, Miller and Shenoy [2] probed the size-dependent elastic properties of nano-plates and beams. Hang et al. [3] explained the size dependent phenomenon by the strain gradient continuum theory. Dingreville et al. [4] investigated the surface free energy and its effect on elastic behavior of the nano-sized particles, wires, and films. Yang [5] studied the size-dependent effective modulus of spherical nanocavities at dilute concentrations. Gao et al. [6] built a simple model to describe the influence of surface stress on the nanoscale adhesive contact. There are a lot of work regarding the surface/interface energy effects on the nanostructures and solids, and we can only include a small part of them here. For more recent developments in this field, the readers can refer to a review article by Wang et al. [7].

To study the mechanical behavior of nano-materials, the most celebrated continuum-based surface/interface model was first established by Gurtin, Murdoch and coworkers [8] [9] [10], which known as the theory of surface elasticity. In the study of nano-scale problems, all material constants appearing in that constitutive model were commonly calibrated with data obtained from either experimental measurements [11] or atomistic simulations [2] [12]. Therefore, the surface effect has been widely adopted to investigate the mechanical phenomena at nano-scale. Cammarata et al. [13] considered the size-dependent deformation in thin film with surface effects. Gao et al. [14] developed a finite-element method to account for the effect of surface elasticity. Wang et al. [15] studied to a half-plane subjected to normal pressures with surface tension. Zhao and Rajapakse [16] proposed a continuum-based model to study the influence of surface stresses on mechanical responses of an elastic half-space compressed by an axisymmetric, rigid, frictionless nano-indentor. Long and Wang [17] studied the effect of the residual surface stress on the two dimensional Hertz contact problem, and later Long et al. [18] generalized their work to the three dimensional case. Wang [19] derived the general analytical solution of nano-contact problem with surface effects by using the complex variable function method. Gao et al. [20] [21] derived the influence of the surface stress on the JKR adhesive contact, which is investigated by employing the non-classical Boussinesq fundamental solutions. In this paper, Fourier integral transform method is used to solve the non-classical boundary value problems with surface effects.

\section{Problem Description}

Now we consider a material occupying the upper half-plane $z>0$, we refer to a Cartesian coordinate system $(o-x y z)$, as shown in Figure 1, where the $x$ axis is along the surface and the $z$ axis perpendicular to the surface. It is assumed that the material is subjected to triangle distribution force $p(x)$ and $q(x)$ over the region $|x| \leq a$. While the normal and shear force form zero $\left(O_{1}\right.$ and $\left.O_{2}\right)$ uniformly increased to maximum $p_{0}$ and $q_{0}$ at the point $O$. The plane-strain conditions are assumed to $\varepsilon_{2 i}=0$, and 


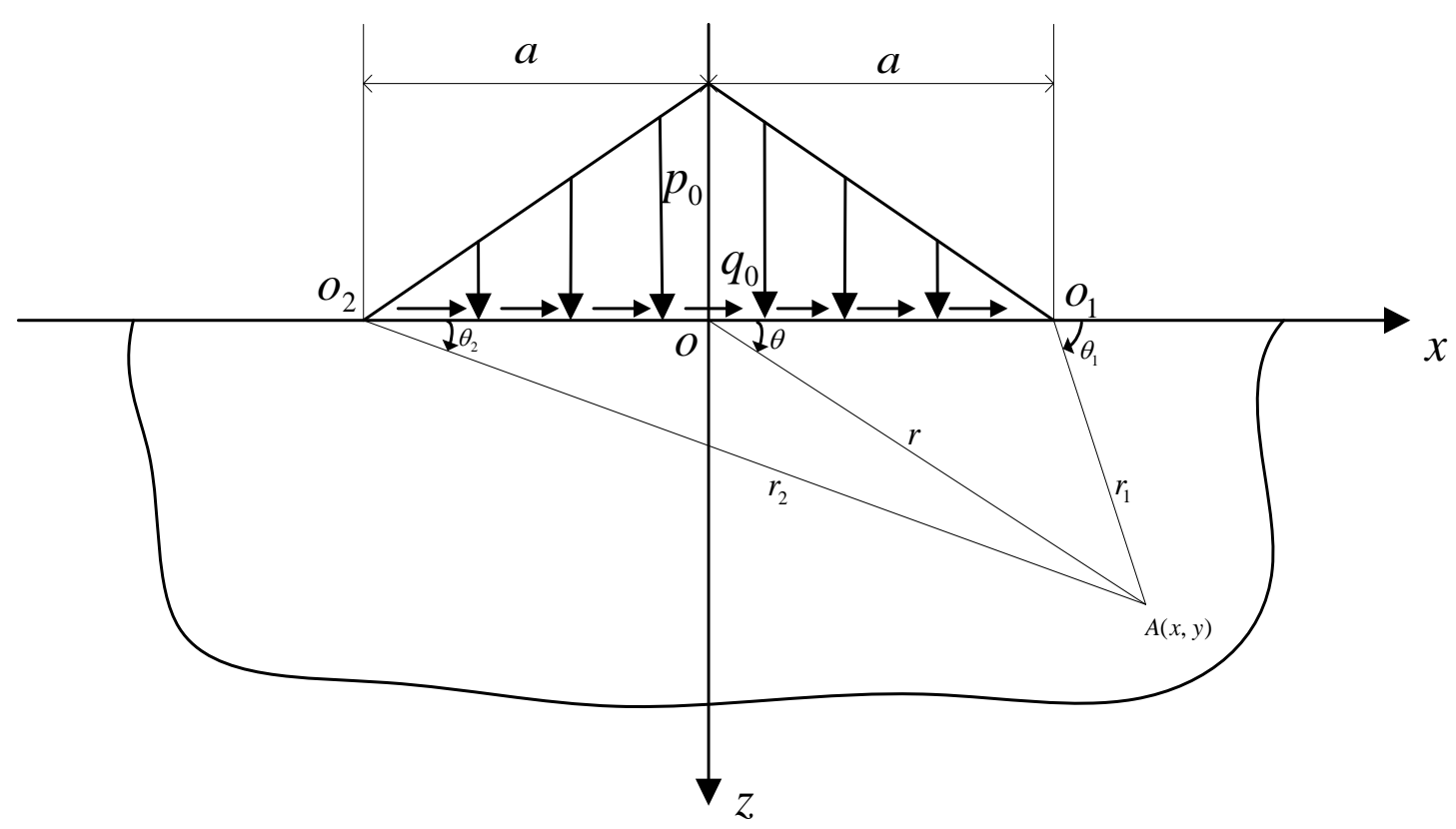

Figure 1. Schematic of contact problem under triangle distribution loads.

the contact is assumed to be frictionless.

The problem statement is to determine the triangle distribution force exerted by the elastic field (e.g., displacement and stresses) with the half-plane for the influence of surface effects.

\section{Basic Equations of Surface Elasticity and General Solutions}

In surface elasticity theory, the equilibrium and constitutive equations in the bulk of material are the same as those in classical elastic theory, but the presence of surface stresses gives rise to a non-classical boundary condition.

\subsection{Basic Equations}

In the absence of body force, the equilibrium equations, constitutive law, and geometry relations in the bulk are as follows

$$
\begin{aligned}
& \sigma_{i j, j}=0 \\
& \sigma_{i j}=2 G\left(\varepsilon_{i j}+\frac{\mu}{1-2 \mu} \varepsilon_{k k} \delta_{i j}\right)
\end{aligned}
$$

where $G$ and $\mu$ are the shear modulus and Poisson's ratio of the bulk material, $\sigma_{i j}$ and $\varepsilon_{i j}$ are the stress tensor and strain tensor in the bulk material, respectively.

The strain tensor is related to the displacement vector $u_{i}$ by

$$
\varepsilon_{i j}=\frac{1}{2}\left(u_{i, j}+u_{j, i}\right)
$$

On the surface, the generalized Young-Laplace equation [22], surface constitutive relation and strain-displacement relationship can be expressed as 


$$
\begin{gathered}
\sigma_{\beta \alpha} n_{\beta}+\sigma_{\beta \alpha, \beta}^{s}=0 \\
\sigma_{i j} n_{i} n_{j}=\sigma_{\beta \alpha}^{s} \kappa_{\beta \alpha} \\
\sigma_{\beta \alpha}^{s}=\tau^{s} \delta_{\beta \alpha}+2\left(\mu^{s}-\tau^{s}\right) \varepsilon_{\beta \alpha}+\left(\lambda^{s}+\tau^{s}\right) \varepsilon_{\gamma \gamma} \delta_{\beta \alpha}+\tau^{s} u_{\beta, \alpha}^{s}
\end{gathered}
$$

where $n_{i}$ denotes the normal to the surface, $\kappa_{\beta \alpha}$ the curvature tensor of the surface, $\sigma_{\alpha \beta}^{\mathrm{s}}$ is the $2 \times 2$ surface stress tensor on the surface, $\sigma_{i j}$ is the stress tensor in the elastic material, and $\varepsilon_{\alpha \beta}^{s}$ is the $2 \times 2$ surface strain tensor which is equal to the tangential components of the strain tensor of the elastic material on the surface, $\tau^{s}$ is the residual surface tension under unstrained conditions, $\mu^{s}$ and $\lambda^{s}$ are surface Lamé constants which can be determined by atom simulations or experiments [2].

\subsection{General Solutions}

Based on previous work by Wang [15] and Wang [23], Fourier integral transformation method is adopted to solve the stress and displacement components by

$$
\begin{aligned}
& \sigma_{x x}=\frac{1}{\sqrt{2 \pi}} \int_{-\infty}^{\infty}[A(\xi)+(z-2|\xi|) B(\xi)] \xi \mathrm{e}^{-i x \xi-z|\xi|} \mathrm{d} \xi \\
& \sigma_{z z}=-\frac{1}{\sqrt{2 \pi}} \int_{-\infty}^{\infty}[A(\xi)+z B(\xi)] \xi^{2} \mathrm{e}^{-i x \xi-z|\xi|} \mathrm{d} \xi \\
& \sigma_{x z}=\frac{i}{\sqrt{2 \pi}} \int_{-\infty}^{\infty} \xi[(1-|\xi| z) B(\xi)-|\xi| A(\xi)] \mathrm{e}^{-i x \xi-z|\xi|} \mathrm{d} \xi \\
& u(x, z)=\frac{i}{2 G \sqrt{2 \pi}} \int_{-\infty}^{\infty}[(2-v)|\xi| A(\xi)+(z|\xi|-2(1-v)) B(\xi)] \mathrm{e}^{-i x \xi-z|\xi|} \mathrm{d} \xi+C_{1} \\
& w(x, z)=\frac{1}{2 G \sqrt{2 \pi}} \int_{-\infty}^{\infty}[|\xi| A(\xi)+(1-2 v+z|\xi|) B(\xi)] \mathrm{e}^{-i x \xi-z|\xi|} \mathrm{d} \xi+C_{2}
\end{aligned}
$$

where $A$ and $B$ are generally functions of $\xi$ as yet to be determined by boundary conditions.

\section{Elastic Solution under Normal Triangle Distribution Force}

As a particular example, let us consider the effect of a normal triangle distribution force $p(x)$ over the region $|x| \leq a$, and the normal force form zero $\left(O_{1}\right.$ and $\left.O_{2}\right)$ uniformly increased to maximum $p_{0}(O)$, while remainder of the boundary $z=0$ being unstressed as shown in Figure 1.

$$
p(x)=\frac{p_{0}}{a}(a-|x|),|x| \leq a
$$

Due to the surface tension mostly influences the normal stress [20]. Therefore, we keep only the first term in Equation (4). Then, the surface stresses are given by

$$
\sigma_{\beta \alpha}^{s}=\tau^{s} \delta_{\beta \alpha}
$$

On the surface, the boundary conditions (3) can be written by

$$
\begin{aligned}
& \sigma_{x z}(x)=0 \\
& p(x)+\sigma_{z z}(x)=-\frac{\tau^{s}}{R(x)}
\end{aligned}
$$


Substituting Equation (8) into Equation (5), one obtains

$$
B=A|\xi|
$$

Due to deformation the radius of curvature of the surface is given by

$$
\frac{1}{R(x)}=\frac{\partial^{2} w(x, 0)}{\partial x^{2}}
$$

By substituting Equations (9) and (10) into the surface condition Equation (8), $A(\xi)$ is determined by

$$
A(\xi)=\frac{\tilde{p}(\xi)}{(1+s|\xi|) \xi^{2}}
$$

where

$$
\begin{gathered}
s=\frac{\tau^{s}(1-v)}{G} \\
\tilde{p}(\xi)=\frac{p_{0}}{a} \sqrt{\frac{2}{\pi}} \frac{1-\cos (a \xi)}{\xi^{2}}
\end{gathered}
$$

where $s$ is a length parameter depending on the surface property and material elastic constants. It should be pointed out that this parameter indicates the thickness size of the zone where the surface effect is significant, and plays a critical role in the surface elasticity. For metals, $s$ is estimated on the order of nanometers.

Therefore $A(\xi)$ is given by

$$
A(\xi)=\frac{p_{0}}{a} \sqrt{\frac{2}{\pi}} \frac{1-\cos (a \xi)}{(1+s|\xi|) \xi^{4}}
$$

Substituting Equation (14) into Equation (5), the stresses component and displaces component are obtained as

$$
\begin{aligned}
& \sigma_{x x}=\frac{2 q_{0}}{\pi a} \int_{0}^{\infty}\left(\frac{z \xi-1}{1+s \xi}\right) \frac{\cos (x \xi)}{\xi^{2}}[1-\cos (a \xi)] \mathrm{e}^{-z \xi} \mathrm{d} \xi \\
& \sigma_{z z}=-\frac{2 q_{0}}{\pi a} \int_{0}^{\infty}\left(\frac{z \xi+1}{1+b \xi}\right) \frac{\cos (x \xi)}{\xi^{2}}[1-\cos (a \xi)] \mathrm{e}^{-z \xi} \mathrm{d} \xi \\
& \sigma_{x z}=-\frac{2 q_{0}}{\pi a} \int_{0}^{\infty}\left(\frac{z}{1+s \xi}\right) \frac{\sin (x \xi)}{\xi}[1-\cos (a \xi)] \mathrm{e}^{-z \xi} \mathrm{d} \xi \\
& u(x, z)=\frac{p_{0}}{\pi G a} \int_{0}^{\infty}\left[\frac{z \xi+2 v-1}{\xi^{3}(1+s \xi)}\right] \sin (x \xi)[1-\cos (a \xi)] \mathrm{e}^{-z \xi} \mathrm{d} \xi+C_{1} \\
& w(x, z)=\frac{p_{0}}{\pi G a} \int_{0}^{\infty} \frac{z \xi+2(1-v)}{\xi^{3}(1+b \xi)} \cos (x \xi)[1-\cos (a \xi)] \mathrm{e}^{-z \xi} \mathrm{d} \xi+C_{2}
\end{aligned}
$$

It is seen, when $s=0$, that is, the surface influence is ignored in Equation (15), the stresses of the half-plane are consistent with those in the classical elastic contact results which is the same result [23], respectively. 


$$
\begin{aligned}
& \sigma_{x x}=\frac{p_{0}}{\pi a}\left[(x-a) \theta_{1}+(x+a) \theta_{2}-2 x \theta+2 z \ln \left(r_{1} r_{2} / r^{2}\right)\right] \\
& \sigma_{z z}=\frac{p_{0}}{\pi a}\left[(x-a) \theta_{1}+(x+a) \theta_{2}-2 x \theta\right] \\
& \sigma_{x z}=-\frac{z p_{0}}{\pi a}\left[\theta_{1}+\theta_{2}-2 \theta\right]
\end{aligned}
$$

where

$$
r_{1}^{2}=(x-a)^{2}+z^{2}, r_{2}^{2}=(x+a)^{2}+z^{2}, r^{2}=x^{2}+z^{2}, \tan \theta_{1}=\frac{z}{x-a}, \tan \theta_{2}=\frac{z}{x+a}, \tan \theta=\frac{z}{x} .
$$

On the contact surface $(z=0)$, the normal stress is given by

$$
\sigma_{z z}(x, 0)=-\frac{2 p_{0}}{\pi} \int_{0}^{\infty}\left(\frac{1-\cos t}{t^{2}}\right)\left(1+\frac{s}{a} t\right)^{-1} \cos \left(\frac{x}{a} t\right) \mathrm{d} t
$$

According to the Saint-Venant's Principle, we assume that the normal displacement is $W$ specified to be zero at a distance $r_{0}$ on the contact surface, that is, $w\left(r_{0}, 0\right)=0$, the displacement on the surface is derived as

$$
w(x, 0)=\frac{2 p_{0}(1-v) a}{\pi G} \int_{0}^{\infty}\left(\frac{1-\cos (t)}{t^{3}}\right)\left(\frac{s}{a}+1\right)^{-1}\left[\cos \left(\frac{x}{a} t\right)-\cos \left(\frac{r_{0}}{a} t\right)\right] \mathrm{d} t
$$

Assuming that the origin has no displacement in the $\mathrm{x}$ direction, that is, $u(0,0)=0$ one obtains

$$
u(x, 0)=-\frac{p_{0}(1-2 v) a}{\pi G} \int_{0}^{\infty}\left(\frac{1-\cos (t)}{t^{3}}\right)\left(\frac{s}{a}+1\right)^{-1} \sin \left(\frac{x}{a} t\right) \mathrm{d} t
$$

As show in Figure 2, the results indicated that the normal stress $\sigma_{z z}$ is a smooth distribution when the surface tension is considered by at the loading boundary $(x= \pm a)$, However, it is just the reverse with classical results that is the surface tension is ignored $(s / a=0)$. In addition, the actual normal stress $\sigma_{\mathrm{zz}}$ is smaller than the classical value in the loading zone and is larger outside of the zone.

Due to the different surface tension value, the horizontal displacement is displayed in Figure 3, where we set $r_{0}=5 a$, and $K_{1}=(1-2 v) / \pi G$. It is seen that the horizontal displacement is continuous everywhere on the deformed surface. However, the classical elasticity theory predicted unreasonably that the horizontal displacement is discontinuously at the load boundary $x= \pm a$, as seen from the curve of $s / a=0$. The indent depth is plotted in Figure 4 with $K_{2}=2(1-v) / \pi G$, which also shows that the slope of the deformed surface is continuous everywhere. It is also found the indent depth decreases with the increase of surface tension.

\section{Elastic Solution under Tangential Triangle Distribution Force}

Now, let us consider the effect of a tangential triangle distribution force $q(x)$ over the region $|x| \leq a$, while the shears force form zero $\left(O_{1}\right.$ and $\left.O_{2}\right)$ uniformly increased to maximum $q_{0}$ at the point $O$, while remainder of the boundary $z=0$ being unstressed as shown in Figure 1. 


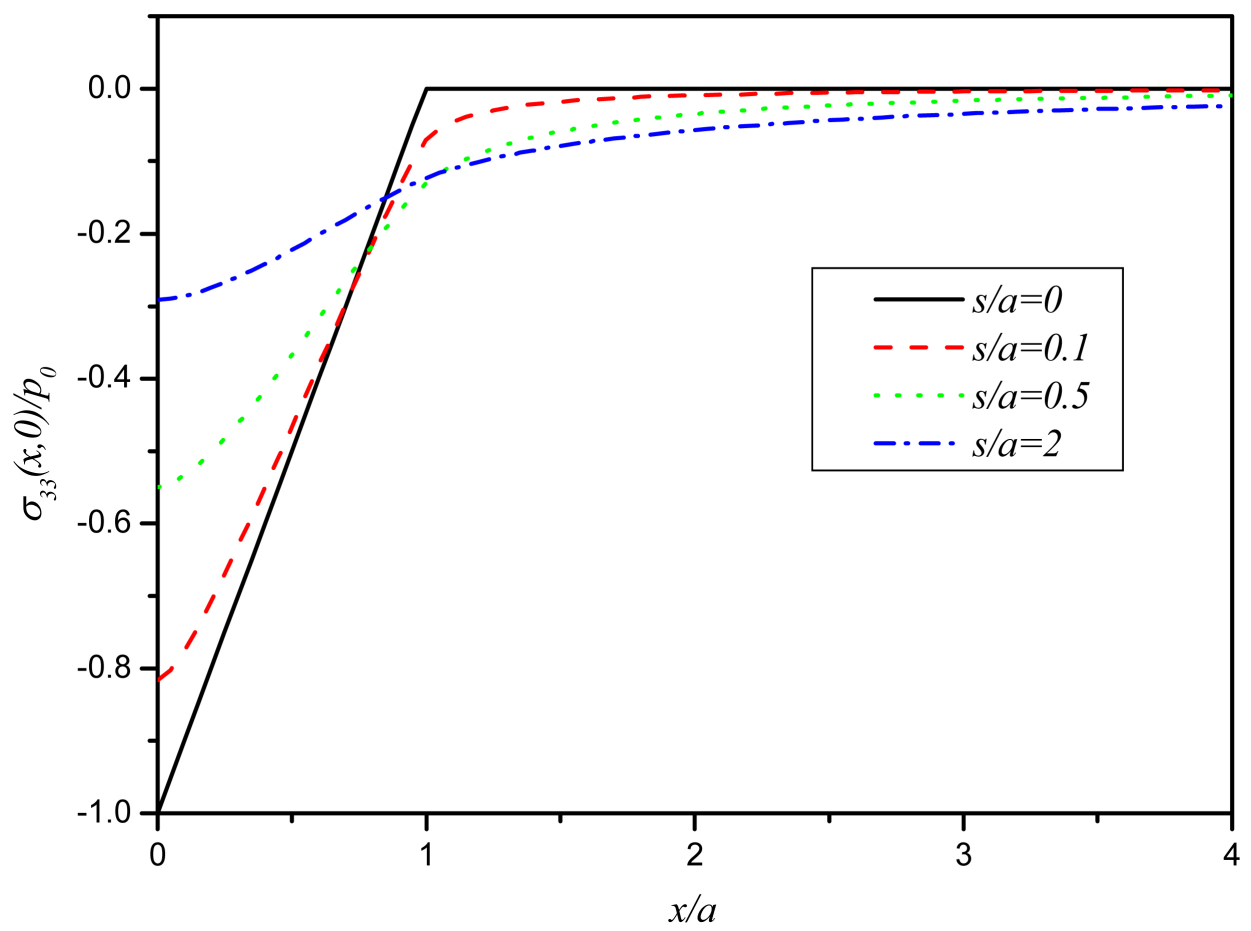

Figure 2. The distribution of contact normal stress $\sigma_{z z}$ under normal triangle distribution load.

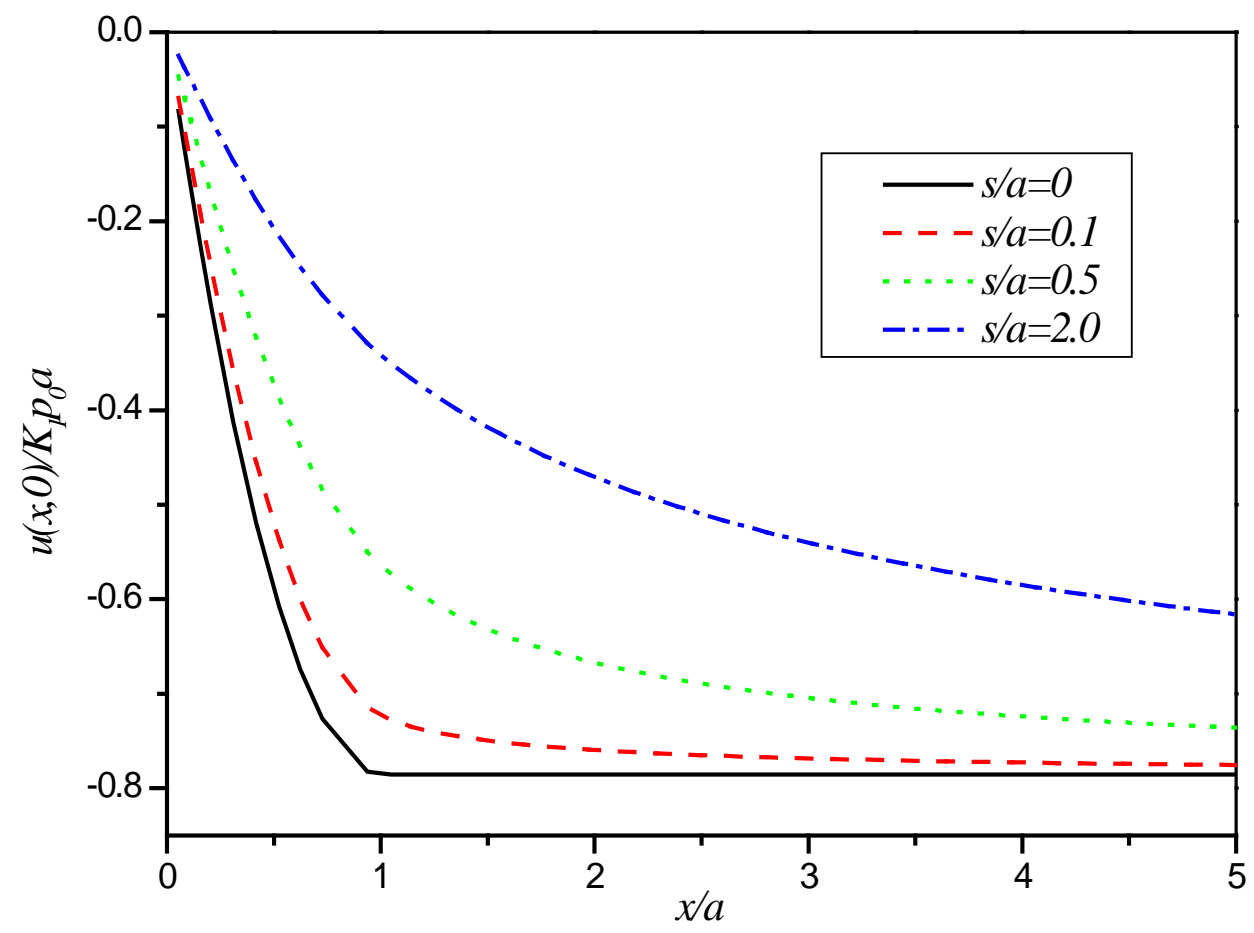

Figure 3. The distribution of surface displacement $u$ under normal triangle distribution load.

At this moment, the boundary conditions (3) on the contact surface $(z=0)$ are simplified to 


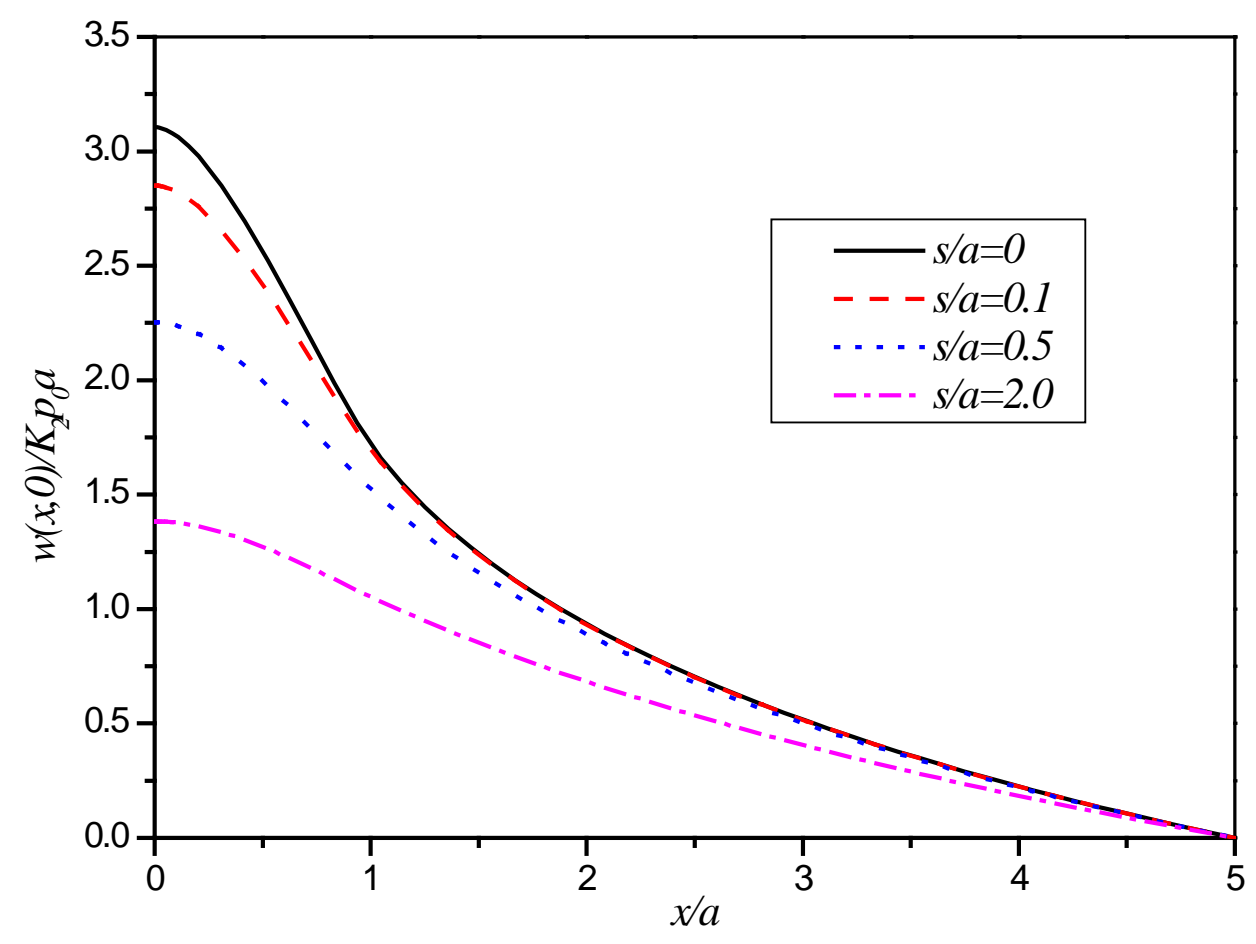

Figure 4. The distribution of surface indentation $w$ under normal triangle distribution load.

$$
\begin{aligned}
& \sigma_{z z}=0 \\
& \sigma_{x z}+q(x)=-\left(\frac{\mathrm{d} \tau^{s}}{\mathrm{~d} x}+k^{s} \frac{\mathrm{d}^{2} u}{\mathrm{~d} x^{2}}\right)
\end{aligned}
$$

where $q(x)$ is the tangential triangle distribution force applied on the materials surface, and $k^{s}=2 \mu^{s}+\lambda^{s}$ is a surface constant.

$$
q(x)=\frac{q_{0}}{a}(a-|x|),|x| \leq a
$$

Substituting Equation (21) into Equation (20), one can be obtained

$$
\begin{aligned}
& A(\xi)=0 \\
& B(\xi)=i \frac{\tilde{q}(\xi)}{\xi} \frac{1}{1+b \xi}
\end{aligned}
$$

where

$$
\begin{gathered}
b=\frac{k^{s}(1-v)}{G} \\
\tilde{q}(\xi)=\frac{1}{\sqrt{2 \pi}} \int_{-\infty}^{\infty} q(x) \mathrm{e}^{i x \xi} \mathrm{d} x
\end{gathered}
$$

where $b$ is a length parameter depending on the material surface property. It should be pointed out that this parameter plays a critical role in the surface elasticity.

Substituting Equation (24) into the surface condition Equations (12) and (13), the solution of stresses and displaces under pure shear load were obtained 


$$
\begin{aligned}
& \sigma_{x x}=\frac{1}{\sqrt{2 \pi}} \int_{-\infty}^{\infty} \xi(z-2|\xi|) B(\xi) \mathrm{e}^{-i x \xi-z|\xi|} \mathrm{d} \xi \\
& \sigma_{z z}=-\frac{1}{\sqrt{2 \pi}} \int_{-\infty}^{\infty} \xi^{2} z B(\xi) \mathrm{e}^{-i x \xi-z|\xi|} \mathrm{d} \xi \\
& \sigma_{x z}=\frac{i}{\sqrt{2 \pi}} \int_{-\infty}^{\infty} \xi(1-|\xi| z) B(\xi) \mathrm{e}^{-i x \xi-z|\xi|} \mathrm{d} \xi \\
& u(x, z)=\frac{i}{2 G \sqrt{2 \pi}} \int_{-\infty}^{\infty}(z|\xi|-2(1-v)) B(\xi) \mathrm{e}^{-i x \xi-z|\xi|} \mathrm{d} \xi+C_{1} \\
& w(x, z)=\frac{1}{2 G \sqrt{2 \pi}} \int_{-\infty}^{\infty}(1-2 v+z|\xi|) B(\xi) \mathrm{e}^{-i x \xi-z|\xi|} \mathrm{d} \xi+C_{2}
\end{aligned}
$$

Substituting Equation (24) into Equations (22), one obtains

$$
\tilde{q}(\xi)=\frac{q_{0}}{a} \sqrt{\frac{2}{\pi}} \frac{1-\cos (a \xi)}{\xi^{2}}
$$

Therefore $B(\xi)$ is given by

$$
B(\xi)=\frac{i q_{0}}{a} \sqrt{\frac{2}{\pi}} \frac{1-\cos (a \xi)}{(1+b \xi) \xi^{3}}
$$

Substituting Equation (27) into Equations (25), the stresses component and displaces component are obtained as

$$
\begin{aligned}
& \sigma_{x x}=\frac{2 q_{0}}{\pi a} \int_{0}^{\infty}\left(\frac{z \xi-2}{1+b \xi}\right) \frac{\sin (x \xi)}{\xi^{2}}[1-\cos (a \xi)] \mathrm{e}^{-z \xi} \mathrm{d} \xi \\
& \sigma_{z z}=-\frac{2 q_{0}}{\pi a} \int_{0}^{\infty}\left(\frac{z}{1+b \xi}\right) \frac{\sin (x \xi)}{\xi}[1-\cos (a \xi)] \mathrm{e}^{-z \xi} \mathrm{d} \xi \\
& \sigma_{x z}=-\frac{2 q_{0}}{\pi a} \int_{0}^{\infty}\left(\frac{1-z \xi}{1+b \xi}\right) \frac{\cos (x \xi)}{\xi^{2}}[1-\cos (a \xi)] \mathrm{e}^{-z \xi} \mathrm{d} \xi \\
& u(x, z)=-\frac{q_{0}}{\pi G a} \int_{0}^{\infty}\left[\frac{z \xi-2(1-v)}{\xi^{3}(1+b \xi)}\right] \cos (x \xi)[1-\cos (a \xi)] \mathrm{e}^{-z \xi} \mathrm{d} \xi+C_{1} \\
& w(x, z)=\frac{q_{0}}{\pi G a} \int_{0}^{\infty} \frac{1-2 v+z \xi}{\xi^{3}(1+b \xi)} \sin (x \xi)[1-\cos (a \xi)] \mathrm{e}^{-z \xi} \mathrm{d} \xi
\end{aligned}
$$

It is seen, when $b=0$, that is, the surface influence is ignored in Equation (28), the stresses and displacements of the half-plane are consistent with those in the classical elastic results [24], respectively.

$$
\begin{aligned}
& \sigma_{x x}=\frac{2 q_{0}}{\pi a}\left[2 x \ln \left(\frac{r_{1} r_{2}}{r^{2}}\right)+2 a \ln \left(\frac{r_{2}}{r_{1}}\right)-3 z\left(\theta_{1}+\theta_{2}-2 \theta\right)\right] \\
& \sigma_{z z}=-\frac{z q_{0}}{\pi a}\left(\theta_{1}+\theta_{2}-2 \theta\right) \\
& \sigma_{x z}=\frac{q_{0}}{\pi a}\left[(x-a) \theta_{1}+(x+a) \theta_{2}-2 x \theta+2 z \ln \left(\frac{r_{1} r_{2}}{r^{2}}\right)\right]
\end{aligned}
$$

On the contact surface $z=0$, the stresses is given by 


$$
\begin{aligned}
& \sigma_{x x}(x, 0)=-\frac{4 q_{0}}{\pi} \int_{0}^{\infty}\left(\frac{1-\cos t}{t^{2}}\right)\left(1+\frac{b}{a} t\right)^{-1} \sin \left(\frac{x}{a} t\right) \mathrm{d} t \\
& \sigma_{x z}(x, 0)=-\frac{2 q_{0}}{\pi} \int_{0}^{\infty}\left(\frac{1-\cos t}{t^{2}}\right)\left(1+\frac{b}{a} t\right)^{-1} \cos \left(\frac{x}{a} t\right) \mathrm{d} t
\end{aligned}
$$

Based on the previous assumption, $u\left(r_{0}, 0\right)=0$, the displacement on the surface component is derived as

$$
\begin{aligned}
& u(x, 0)=\frac{2(1-v) q_{0} a}{\pi G} \int_{0}^{\infty}\left(\frac{1-\cos t}{t^{3}}\right)\left(1+\frac{b}{a} t\right)^{-1}\left[\cos \left(\frac{x}{a} t\right)-\cos \left(\frac{r_{0}}{a} t\right)\right] \mathrm{d} t \\
& w(x, 0)=\frac{(1-2 v) q_{0} a}{\pi G} \int_{0}^{\infty}\left(\frac{1-\cos t}{t^{3}}\right)\left(1+\frac{b}{a} t\right)^{-1} \sin \left(\frac{x}{a} t\right) \mathrm{d} t
\end{aligned}
$$

It is instructive to examine the influence of the surface elasticity on the stresses and displacements of the contact surface and compare them with those in classical contact problem. Figure 5 and Figure 6 show the distribution of the stresses $\sigma_{x x}$ and $\sigma_{x z}$ on the contact surface, where the solution of $b / a=0$ is consistent with the classical elastic result.

It can be seen from Figure 7 that the normal stress $\sigma_{x x}$ transits continuously across the loading boundary $x= \pm a$, which is increasing monotonically with respect to $\mathrm{x}$ in the loading region $(|x / a|<0.75)$, and the inverse is observed outside the loading region $(|x / a|>0.75)$. It means that the analytical expressions can give approximate results in the region that is far from the loading boundary. It is also found Figure 5 that shear stress changes smoothly across the loading boundary $x= \pm a$, which is different from a

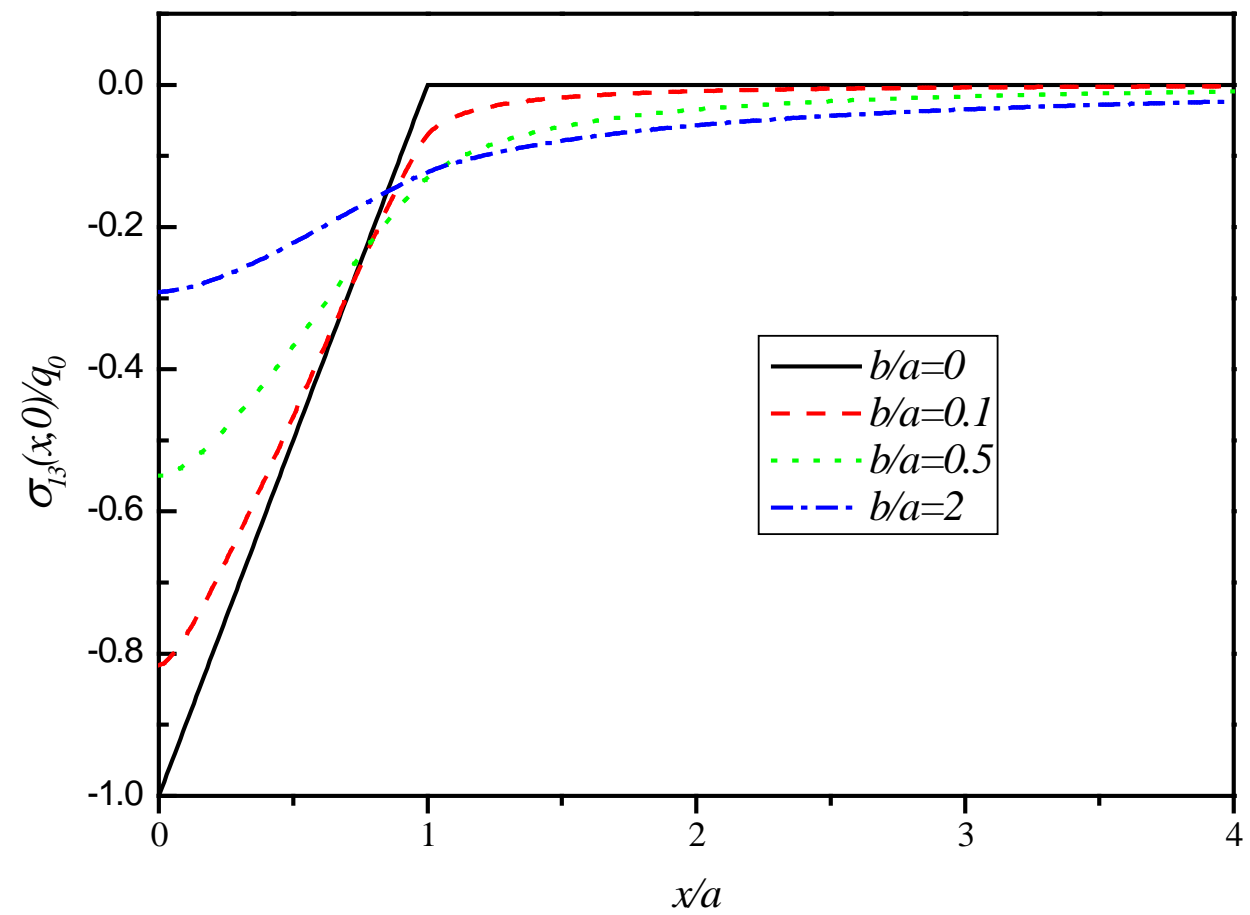

Figure 5. The distribution of the shear stress $\sigma_{x z}$ under tangential triangle distribution load. 


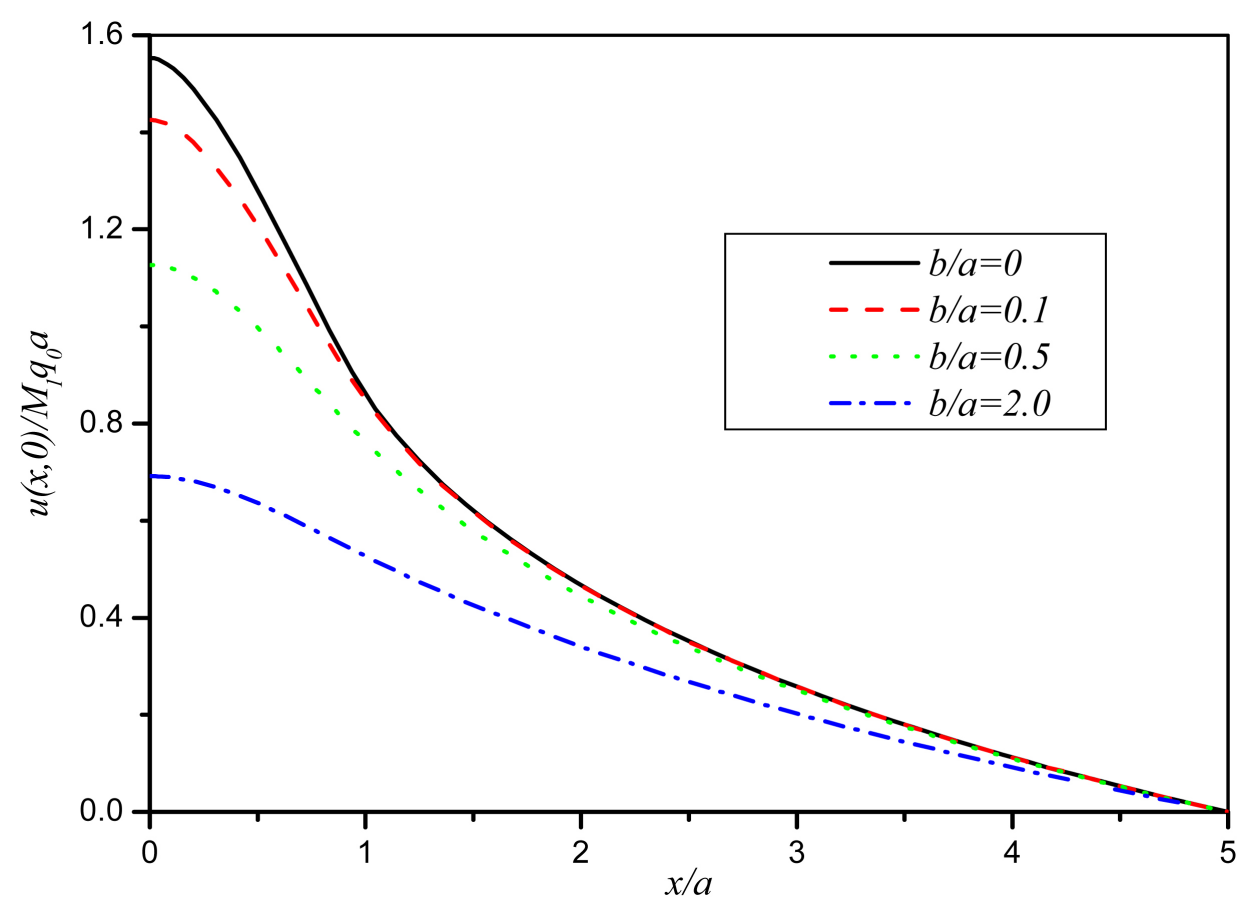

Figure 6. The distribution of surface displacement $u$ under tangential triangle distribution load.

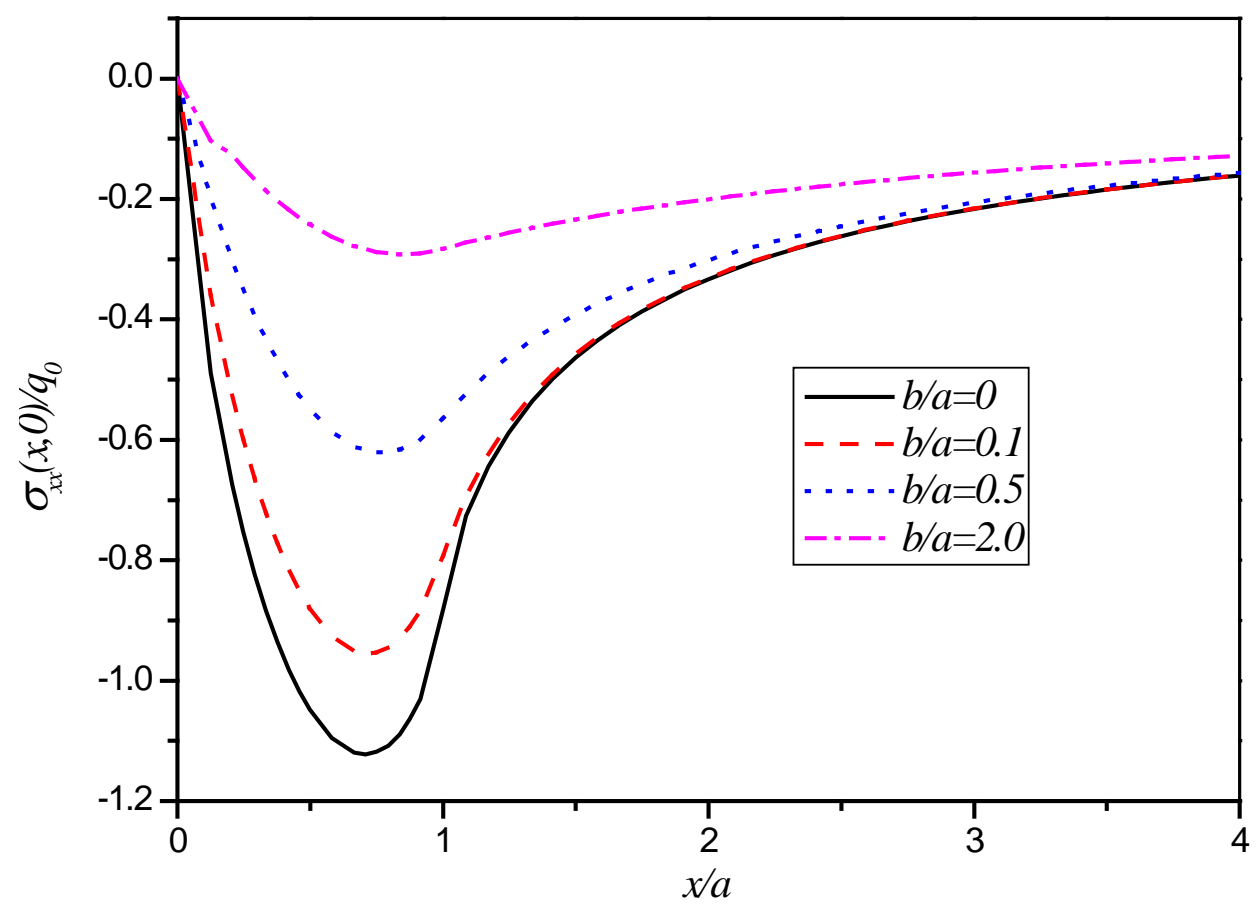

Figure 7. The distribution of the normal stress $\sigma_{x x}$ under tangential triangle distribution load.

singularity predicted by classical elasticity.

Due to the different surface elasticity value, the horizontal displacement is displayed in Figure 6, where we set $r_{0}=5 a$, and $M_{1}=2(1-v) / \pi G$. It is seen that the slope of 


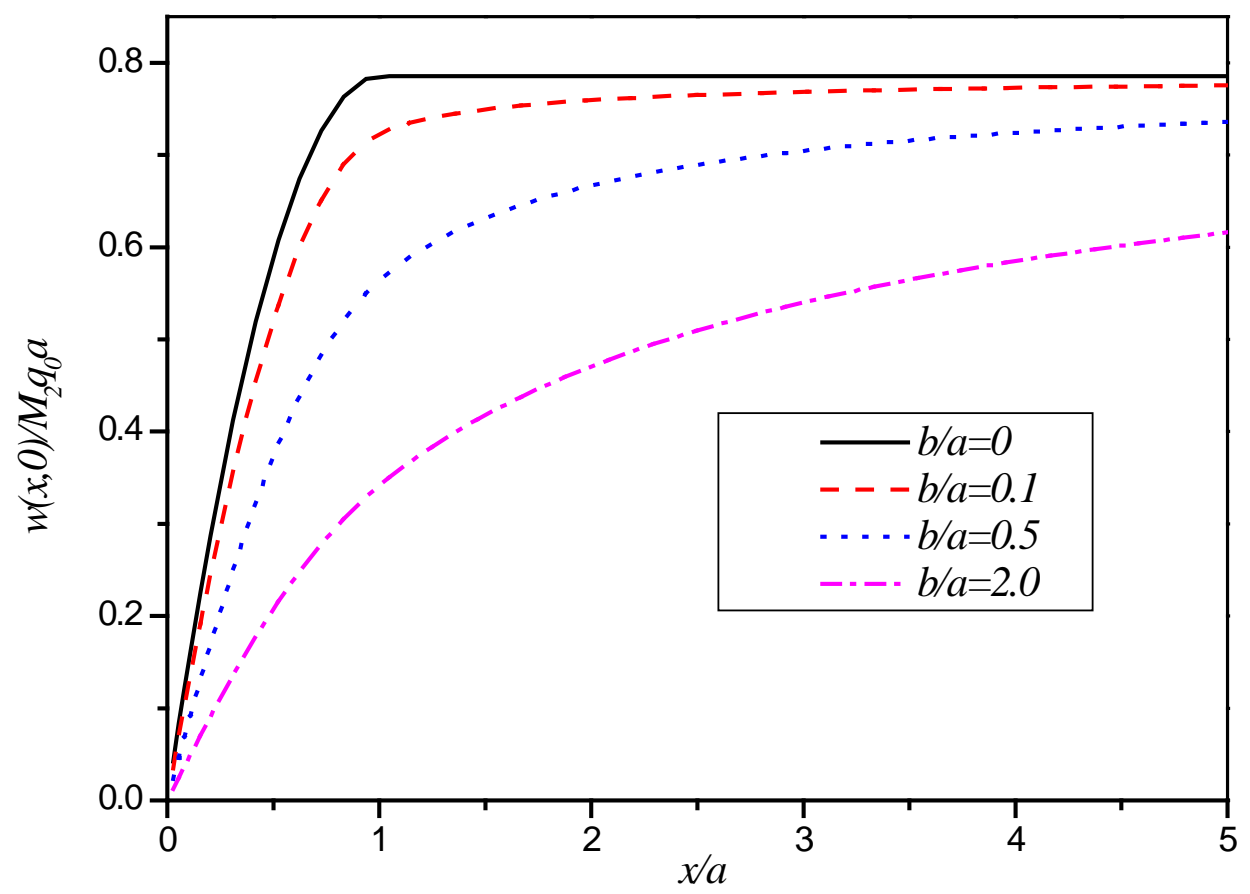

Figure 8. The distribution of surface indentation $w$ under tangential triangle distribution load.

the deformed surface for $a>0$ is continuous everywhere. It is also found the horizontal displacement decreases with the increase of surface elasticity. The indent depth is plotted in Figure 8 with $M_{2}=(1-2 v) / \pi G$, which also shows that the normal displacement is continuous everywhere on the deformed surface. In addition, the indent depth decreases continuously with the increase of surface elasticity.

\section{Conclusion}

In this paper, we consider the two-dimensional contact problem in the light of surface elasticity theory. Fourier integral transform method is adopted solving general analytical solution. For two particular loading cases of triangle distribution forces, the results are analyzed in detail and compared with the classical linear elastic solutions. A series of theoretical and numerical results show that the influences of the surface tension and the surface elasticity on the stresses and displacements are not always equal. It is found that the surface elasticity theory illuminates some interesting characteristics of contact problems at nano-scale, which are distinctly different from the classical solutions of elasticity without surface effects. Therefore, the influence of surface effects should be considered for nano-contact problems.

\section{References}

[1] Cammarata, R.C. (1994) Surface and Interface Stress Effects in Thin Films. Progress in Surface Science, 46, 1-38. http://dx.doi.org/10.1016/0079-6816(94)90005-1

[2] Miller, R.E. and Shenoy, V.B. (2000) Size-Dependent Elastic Properties of Nanosized Structural Elements. Nanotechnology, 11, 139-147. 
http://dx.doi.org/10.1088/0957-4484/11/3/301

[3] Huang, Y., Zhang, F. and Hwang, K.C. (2006) A Model of Size Effects in Nano-Indentation. Journal of the Mechanics \& Physics of Solids, 54, 1668-1686.

http://dx.doi.org/10.1016/j.jmps.2006.02.002

[4] Dingreville, R., Qu, J. and Cherkaoui, M. (2005) Surface Free Energy and Its Effect on the Elastic Behavior of Nano-Sized Particles, Wires And films. Journal of the Mechanics \& Physics of Solids, 53, 1827-1854. http://dx.doi.org/10.1016/j.jmps.2005.02.012

[5] Yang, F. (2004) Size-Dependent Effective Modulus of Elastic Composite Materials: Spherical Nanocavities at Dilute Concentrations. Journal of Applied Physics, 95, 3516-3520. http://dx.doi.org/10.1063/1.1664030

[6] Gao, X., Hao, F. and Huang, Z. (2014) Mechanics of Adhesive Contact at the Nanoscale: The Effect of Surface Stress. International Journal of Solids \& Structures, 51, 566-574. http://dx.doi.org/10.1016/j.ijsolstr.2013.10.017

[7] Wang, J.X., Huang, Z.P., et al. (2011) Surface Stress Effect in Mechanics of Nanostructured Materials. Acta Mechanica Solida Sinica, 24, 52-82.

http://dx.doi.org/10.1016/s0894-9166(11)60009-8

[8] Gurtin, M.E. and Murdoch, A.I. (1975) A Continuum Theory of Elastic Material Surfaces. Archive for Rational Mechanics \& Analysis, 57, 291-323. http://dx.doi.org/10.1007/BF00261375

[9] Gurtin, M.E. and Murdoch, A.I. (1976) Effect of Surface Stress on Wave Propagation in Solids. Journal of Applied Physics, 47, 4414-4421. http://dx.doi.org/10.1063/1.322403

[10] Gurtin, M.E. and Murdoch, A.I. (1978) Surface Stress in Solids. International Journal of Solids \& Structures, 14, 431-440. http://dx.doi.org/10.1016/0020-7683(78)90008-2

[11] Jing, G.Y., Duan, H.L., Sun, X.M., et al. (2006) Surface Effects on Elastic Properties of Silver Nanowires: Contact Atomic-Force Microscopy. Physical Review B, 73, 235409. http://dx.doi.org/10.1103/physrevb.73.235409

[12] Shenoy, V.B. (2005) Atomistic Calculations of Elastic Properties of Metallic Fcc Crystal Surfaces. Physical Review B, 71, 4104. http://dx.doi.org/10.1103/PhysRevB.71.094104

[13] Cammarata, R.C., Sieradzki, K. and Spaepen, F. (2000) Simple Model for Interface Stresses with Application to Misfit Dislocation Generation in Epitaxial Thin Films. Journal of Applied Physics, 87, 1227-1234. http://dx.doi.org/10.1063/1.372001

[14] Wei, G., Shouwen, Y. and Ganyun, H. (2014) Finite Element Characterization of the SizeDependent Mechanical Behaviour in Nanosystems. Nanotechnology, 17, 1118-1122. http://dx.doi.org/10.1088/0957-4484/17/4/045

[15] Wang, G.F. and Feng, X.Q. (2007) Effects of Surface Stresses on Contact Problems at Nanoscale. Journal of Applied Physics, 101, 013510. http://dx.doi.org/10.1063/1.2405127

[16] Zhao, X.J. and Rajapakse, R.K.N.D. (2009) Analytical Solutions for a Surface-Loaded Isotropic Elastic Layer with Surface Energy Effects. International Journal of Engineering Science, 47, 1433-1444. http://dx.doi.org/10.1016/j.ijengsci.2008.12.013

[17] Long, J.M., Wang, G.F. and Feng, X.Q. (2012) Two-Dimensional Hertzian Contact Problem with Surface Tension. International Journal of Solids \& Structures, 49, 1588-1594. http://dx.doi.org/10.1016/j.ijsolstr.2012.03.017

[18] Long, J.M. and Wang, G.F. (2013) Effects of Surface Tension on Axisymmetric Hertzian Contact Problem. Mechanics of Materials, 56, 65-70. http://dx.doi.org/10.1016/j.mechmat.2012.09.003

[19] Lei, D.X., Wang, L.Y. and Ou, Z.Y. (2012) Elastic Analysis for Nanocontact Problem with 
Surface Stress Effects under Shear Load. Journal of Nanomaterials, 6, 4531-4531.

http://dx.doi.org/10.1155/2012/505034

[20] Gao, X., Hao, F., Fang, D., et al. (2013) Boussinesq Problem with the Surface Effect and Its Application to Contact Mechanics at the Nanoscale. International Journal of Solids \& Structures, 50, 2620-2630. http://dx.doi.org/10.1016/j.ijsolstr.2013.04.007

[21] Gao, X., Hao, F., Fang, D., et al. (2014) Mechanics of Adhesive Contact at the Nanoscale: The Effect of Surface Stress. International Journal of Solids \& Structures, 51, 566-574.

http://dx.doi.org/10.1016/j.ijsolstr.2013.10.017

[22] Povstenko, Y.Z. (1993) Theoretical Investigation of Phenomena Caused by Heterogeneous Surface Tension in Solids. Journal of the Mechanics \& Physics of Solids, 41, 1499-1514. http://dx.doi.org/10.1016/0022-5096(93)90037-G

[23] Wang, L.Y. (2015) Elastic Analysis for Contact Problems with Surface Effects under Normal Load. Mathematics \& Mechanics of Solids. http://dx.doi.org/10.1177/1081286514568585

[24] Johnson, K.L. (1985) Contact Mechanics. Cambridge University Press, London. http://dx.doi.org/10.1017/CBO9781139171731

Submit or recommend next manuscript to SCIRP and we will provide best service for you:

Accepting pre-submission inquiries through Email, Facebook, LinkedIn, Twitter, etc. A wide selection of journals (inclusive of 9 subjects, more than 200 journals)

Providing 24-hour high-quality service

User-friendly online submission system

Fair and swift peer-review system

Efficient typesetting and proofreading procedure

Display of the result of downloads and visits, as well as the number of cited articles Maximum dissemination of your research work

Submit your manuscript at: http://papersubmission.scirp.org/

Or contact jamp@scirp.org 